\title{
Reference materials for chemical analysis
}

\author{
Stephen A. Wise • Hendrik Emons
}

Published online: 19 February 2015

(C) Springer-Verlag Berlin Heidelberg (outside the USA) 2015

\begin{abstract}
A journal devoted to publishing advances in analytical and bioanalytical chemistry, such as Analytical and Bioanalytical Chemistry (ABC), generally focuses on reporting new analytical methods or the application of methods to new analytical problems. Frequently, the necessary concepts and 'tools' for producing analytical data that are sufficiently reliable for making important decisions, in addition to satisfying scientific curiosity, are somewhat undervalued. This is also demonstrated in various papers reporting new analytical methods when it comes to method validation. There are still analytical communities that focus such method validation studies only on the characterization of the achievable method precision, but do not consider a suitable evaluation of the trueness of the results. It is recognized that it can be very challenging to assess the latter when appropriate certified reference materials (CRM) are not available to assist in the validation process. Therefore, this special paper collection is intended to address a number of the current challenges regarding "Reference Materials for Chemical Analysis".

Building on a legacy initiated with Fresenius' Zeitschrift für Analytische Chemie (later called Fresenius' Journal of Analytical Chemistry), $\mathrm{ABC}$ has had a long association with
\end{abstract}

Published in the topical collection Reference Materials for Chemical Analysis with guest editors Hendrik Emons and Stephen A. Wise.

S. A. Wise $(\triangle)$

Chemical Sciences Division, National Institute of Standards and

Technology (NIST), Gaithersburg, MD 20899, USA

e-mail: stephen.wise@nist.gov

H. Emons

Joint Research Centre, European Commission, Institute for

Reference Materials and Measurements (IRMM),

2440 Geel, Belgium

e-mail: Hendrik.EMONS@ec.europa.eu papers related to the production and use of reference materials. Since 1987, collections of papers concerning reference materials had been associated with papers presented at the International Symposium for Biological and Environmental Reference Materials (BERM). For this topical collection on reference materials, however, we specifically invited contributions from producers of CRMs worldwide, primarily from the National Institutes of Metrology (NMIs) in various countries. The response to our invitation was enthusiastic and overwhelming with 57 papers submitted. This topical collection illustrates the global involvement in the development of reference materials with contributions from authors working in 17 countries, the European Commission, and the Bureau International des Poids et Mesures (BIPM). The metrology institutes contributing to this paper collection include: Institute for Reference Materials and Measurements (IRMM), Federal Institute for Materials Research and Testing (BAM) in Germany, Physikalisch-Technische Bundesanstalt (PTB) in Germany, LGC Limited (LGC) in the UK, Laboratoire National de Métrologie et d'Essais (LNE) in France, National Metrology Institute of Japan (NMIJ), National Institute of Metrology (NIM) in China, National Measurement Institute Australia (NMIA), Government Laboratory Hong Kong (GLHK), Korea Research Institute for Science and Standards (KRISS), National Metrology Institute of South Africa (NMISA), National Institute of Metrology, Quality and Technology (INMETRO) in Brazil, National Research Council of Canada (NRCC), and the National Institute of Standards and Technology (NIST) in USA. International cooperation among metrology institutes and other government and academic organizations to produce CRMs is illustrated by several papers (e.g., H. Emteborg et al., L. Wu et al.).

This impressive number of manuscript submissions covers a broad range of topics related to CRMs, from their 
development and production to advances in material characterization and application. Because of the scope of Analytical and Bioanalytical Chemistry, only submissions with a strong emphasis on new and/or novel analytical aspects related to reference material development and use were accepted through the journal review process. Analytical "novelty" for reference material papers may include: (1) development of new analytical methods for use in the assignment of values, (2) new materials/matrices as CRMs and/or new analytes in CRMs, (3) novel approaches for CRM preparation, and (4) the combination and comparison of results from different analytical techniques to assign values. Papers in this issue address the following areas related to reference materials:

- New reference material developments (i.e., new materials/ matrices and new analytes);

- Novel approaches for preparing candidate reference materials;

- Novel analytical methods for characterization measurements;

- New analytical methods with high accuracy (trueness and/ or precision) for characterizing candidate CRMs, purity assessments, or stability monitoring.

The largest number of papers in this topical collection addresses new developments in reference materials (both new matrices and new analytes) for environmental, industrial, clinical, food, nutritional, and forensic analysis. J. Reiner and colleagues from NIST and other government and academic laboratories describe the assignment of values for polyfluorinated substances in existing sediment, soil, house dust, and sewage sludge CRMs through an interlaboratory study. The development of a new CRM for azaspiracids (marine biotoxins) in mussel tissue is the subject of the paper by $\mathrm{P}$. McCarron and coworkers from NRCC. Papers from BAM describe the development of two new CRMs: trichothecene mycotoxins (T-2 and HT-2) as the primary toxins in a cereal CRM (R. Köppen et al.) and a CRM for total cyanide in soil with operationally-defined values assigned based on an analytical method (H. Scharf and W. Bremser). T. Roth and colleagues at the University of Erlangen-Nuremberg in Germany report the production of a styrene-based polymer reference material containing phosphorus-based flame retardants. The first CRM for metabolites of organic contaminants, including hydroxylated polycyclic aromatic hydrocarbons, phthalate metabolites, phenols, and volatile organic compound metabolites, in smoker and non-smoker urine is described by M. Schantz with colleagues at NIST and the U.S. Centers for Disease Control and Prevention (CDC). D. Sin and colleagues at the GLHK have developed a green tea-matrix CRM with certified values for alpha- and beta-endosulfan and endosulfan sulfate based on measurements using two isotope dilution (ID) mass spectrometry (MS) procedures. L. Neves et al. from INMETRO (Brazil) describe the development of a CRM for volatile organics (BTEX - benzene, toluene, ethylbenzene and xylenes) to assure the quality of measurements for these contaminants in the environment. P. Fisicaro and colleagues at LNE describe the development of a CRM for toxic metal content $(\mathrm{Ag}, \mathrm{Cd}, \mathrm{Ni}$, and $\mathrm{Pb})$ in atmospheric particles $(10 \mu \mathrm{m})$ deposited on quartz filters. To meet the needs of forensic laboratories in South Africa, M. Archer and coworkers at NMISA describe the preparation and certification process for a CRM with a high concentration of aqueous sodium fluoride using classical complexometric methods and primary titrimetry. Two new CRMs developed by BAM for the characterization of the performance of surface analytical instruments are described by V. Rackwitz et al. and M. Senomer et al.

The importance of the preparation process for reference materials to achieve the required homogeneity and stability is often overlooked. H. Emteborg and colleagues at IRMM, BAM, PTB, and LGC collaborated on a novel approach to address the challenges associated with preparing and maintaining a whole water reference material for the determination of priority pollutants. Comparison of different material preparation approaches (e.g., freeze drying, freezing, autoclaving) for producing food-matrix CRMs for the determination of pesticides (cucumber matrix) and polycyclic aromatic hydrocarbons (baby food material) are discussed in two papers by M. Dabrio Ramos and colleagues at IRMM with recommendations as to the suitability of the various materials with regard to homogeneity and stability. Almost all measurements in inorganic analysis are based on gravimetrically prepared elemental reference solutions. A. Kaltenbach and colleagues at PTB describe the challenging case of preparing such reference solutions for the difficult elements molybdenum and rhodium. M. Nohr et al. (BAM) discuss novel approaches for the eventual development of a reference material to support indoor measurements of volatile organic compounds emitted from building materials.

New and novel analytical methods and/or measurement approaches are often developed as part of the process to assign certified property values to a reference material. Assessment of the purity is a critical aspect of chemical CRM development. Two papers focus on the use of quantitative nuclear magnetic resonance spectroscopy (qNMR) for purity assessment of organic substances. S. Davies and colleagues at NMIA examined the use of qNMR for purity assessment of organic reference standards and demonstrated equivalence between purity values assigned by the traditional mass balance approach and qNMR. M. Weber et al. (Sigma-Aldrich, Switzerland) used a novel qNMR approach to achieve traceability for qNMR measurements for purity assessment of phosphorus-containing compounds. L. Wu et al., as part of a collaboration of three Asian NMIs (KRISS, NIM, and NMIJ), report the purity assessment of a porcine insulin CRM using a combination of techniques including ID-LCMS. M. Kato and NMIJ colleagues developed a C-reactive 
protein solution CRM with the certified value assigned based on combining results from two independent hydrolysis and ID-LC-MS and MS/MS methods. R. Joseph et al. (BIPM) developed a normal-phase LC-MS/MS method with atmospheric pressure photoionization (APPI) for the determination of 10 estrogenic steroid hormones and applied it for purity assessment of a 17ß-estradiol candidate reference material used in an interlaboratory study among NMIs. N. Hanari and coworkers at NMIJ describe the certification of five high-purity CRMs for alkylphenols.

M. Phillips (NIST) presents a new LC-MS/MS method for the simultaneous determination of $\mathrm{B}$ vitamins in different food-matrix CRMs including infant formula, milk powder, egg powder, soy flour, baking chocolate, peanut butter, baby food composite, breakfast cereal, meat homogenate, and blueberries. A two-dimensional heart-cut LC-LC method is reported by A. Breidbach and F. Ulberth (IRMM) for high accuracy aflatoxin $\mathrm{B}_{1}$ measurements for proficiency testing samples (cereal baby food, maize, and maize-based feed) as part of the activities of the EU Reference Laboratory for Mycotoxins. P. Dunn et al. (LGC) describe novel strategies for the determination of the stable carbon absolute isotope ratio of a glycine candidate reference material using elemental analyser-isotope ratio MS. C. Pfeiffer and colleagues at CDC developed an LC-MS/MS method for the determination of methylmalonic acid in human serum to monitor the vitamin $B_{12}$ status in population studies; the results were compared to a previously used CDC GC-MS method and a NIST GC-MS method used to assign a methylmalonic acid value in a serum CRM. U. Resch-Genger and coworkers at BAM present the development of a novel calibraton test slide for a commercial DNA-based assay platform that consists of precisely arranged fluorescent areas of differing intensities; the eventual use of this slide would be to provide a fluorescence intensity scale for referencing of assay results to improve comparability of diagnostic tests.

The challenges associated with maintaining and monitoring long-term stability of CRMs are addressed in papers by $\mathrm{F}$. Gonzaga and J. Dias (INMETRO) for $\mathrm{pH}$ measurements (10 to 21 months), K. Pratt (NIST) for consistency of $\mathrm{pH}$ measurements in CRMs over 70 years, and M. Schantz et al. (NIST) for polycyclic aromatic hydrocarbons and polychlorinated biphenyls in frozen mussel tissue CRMs over 25 years.
This special collection could be realized only because of the enthusiasm and willingness of all the contributing authors to prepare and submit their manuscripts. As the Guest Editors, we would like to thank the authors, the numerous reviewers, and the Editorial Office team of Analytical and Bioanalytical Chemistry for the exceptional cooperation in producing this topical collection.

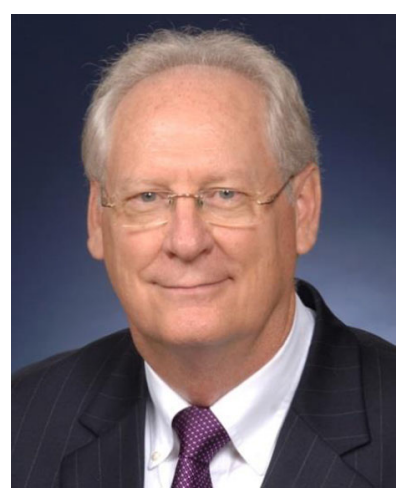

Stephen A. Wise is Senior Analytical Chemist in the Chemical Sciences Division of the National Institute of Standards and Technology (NIST) in Gaithersburg, Maryland, USA. During his career at NIST, he has been primarily involved in the development of certified reference materials for trace organic constituents in environmental, clinical, food, and dietary supplement matrices. $\mathrm{He}$ is currently an Editor of Analytical and Bioanalytical Chemistry, Topical Editor for Analytical Separation Techniques for Polycyclic Aromatic Compounds, and a member of the Advisory Board of Accreditation and Quality Assurance.

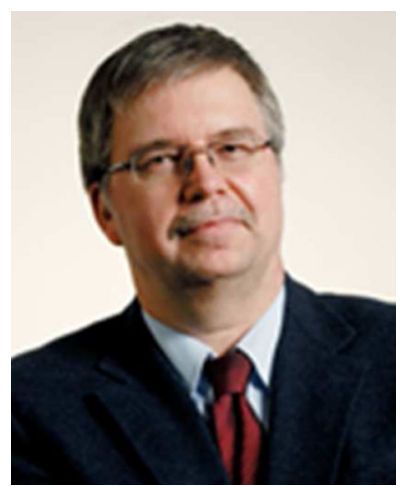

Hendrik Emons is Head of the Unit 'Standards for Innovation and Sustainable Development' of the European Commissions' Joint Research Centre, Institute for Reference Materials and Measurements (IRMM), located in Geel, Belgium. He is in charge of programmes on the development and provision of measurement standards, in particular reference materials, for a broad range of application areas from microbiological to environmental analysis, from biotechnology (including GMOs) and clinical analysis to engineered materials and nanotechnology. He is, moreover, Associate Professor at the University of Duisburg-Essen, Germany, and is on various scientific advisory boards and committees. $\mathrm{He}$ is the Editor-in-Chief of Accreditation and Quality Assurance and serves also on the Advisory Board of Analytical and Bioanalytical Chemistry. 